ARTICLE OPEN

\title{
Role of atomic-scale thermal fluctuations in the coercivity
}

\author{
Yuta Toga $\mathbb{D}^{1,2,3 凶}$, Seiji Miyashita ${ }^{2,3,4}$, Akimasa Sakuma ${ }^{5}$ and Takashi Miyake $\mathbb{D}^{2,6}$
}

The microscopic mechanism of coercivity at finite temperature is a crucial issue for permanent magnets. Here we present the

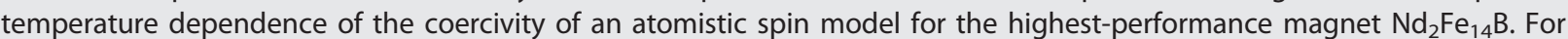
quantitative analysis of the magnetization reversal with thermal fluctuations, we focus on the free energy landscape as a function of the magnetization. The free energy is calculated by the replica-exchange Wang-Landau method. This approach allows us to address a slow nucleation problem, i.e., thermal activation effects, in the magnetization reversal. We concretely observed that the thermal fluctuations lead to a downward convexity in the coercivity concerning the temperature. Additionally, through analyzing the microscopic process of the thermal activation (nucleation), we discover the activation volume is insensitive to a magnetic field around the coercivity. The insensitivity explains the linear reduction of the free energy barrier by the magnetic field in the nucleation process.

npj Computational Materials (2020)6:67; https://doi.org/10.1038/s41524-020-0325-6

\section{INTRODUCTION}

Demands on high-performance permanent magnets have become an imperative subject of the save-energy technology for sustainable environments ${ }^{1,2}$. To improve the performance of magnets, there is often a great desire to understand the temperature dependence of coercivity. Experimental and theoretical researches have been extensively carried out to investigate the microscopic understanding of the coercivity mechanism from atomic-scale magnetic structure ${ }^{3-10}$. Recently, a scanning hard X-ray microprobe was employed to visualize the nucleation and domain wall motion of magnetization reversal processes from the grain boundary interfaces in the highest performance magnet $\mathrm{Nd}_{2} \mathrm{Fe}_{14} \mathrm{~B}^{8}$. Until now, a study on the microscopic approach to temperature effects on coercivity, especially a slow nucleation process, has not been reported. The nucleation leads to the observation time dependence of the coercivity. This is phenomenologically known as coercivity reduction by thermal activation effect $^{11-15}$

Large reduction of coercivity from the theoretically expected value is an essential current problem which has been called "Discrepancy to theory" by Kronmüller et al. $^{16}$. In this regard, extensive works have been done to elucidate the reason for the discrepancy ${ }^{17-21}$, but not yet clear from the atomistic viewpoint. The present paper studies the reduction due to thermal fluctuations. The magnetization reversal is a transition from a metastable state to a stable state by overcoming energy barriers under a reverse magnetic field. In this overcoming process, a reverse nucleus is formed. Under thermal fluctuations, the nucleation occurs stochastically, that is, thermal-activated relaxation. Thus the coercivity, i.e., the threshold field, depends on the observation time. In the permanent magnet applications, the metastable state is required to be stable for the duration of the order of a second. To handle such a slow relaxation process, a method that uses the energy landscape has been developed, also known as the minimum energy path (MEP) ${ }^{22}$. For the magnetization reversal, MEP has been studied in the continuum approximation models with parameters with respect to a given temperature ${ }^{15,18,19,23}$. However, the aforementioned method has not considered thermal fluctuations. In the present work, we propose an approach to evaluate the temperature dependence of the coercivity from a microscopic viewpoint by using an atomistic model. In contrast to the MEP, the present technique directly handles thermal fluctuations and also the atomistic information in the magnetization reversal process. In particular, we focus on the Neodymium (Nd) magnet $\mathrm{Nd}_{2} \mathrm{Fe}_{14} \mathrm{~B}^{24}$.

The coercivity is a non-equilibrium concept concerning the collapse of a metastable state, but not expressed by an equilibrium expectation value. Thus, we need a dynamical model. The relaxation time is estimated from the free-energy barrier $F_{\mathrm{B}}$ by using the form (like Arrhenius law):

$\tau=\tau_{0} \mathrm{e}^{\beta F_{\mathrm{B}}}$,

where $\tau_{0}$ is the reciprocal of the attempt frequency, which is typically set to $10^{-11} \mathrm{~s}^{12}$. Then, for the observation time of $1 \mathrm{~s}$, setting $\tau=1 \mathrm{~s}$, the barrier height is given by $F_{\mathrm{B}}=25.3 k_{\mathrm{B}} T$. The free energy is given by the $\mathrm{Nd}_{2} \mathrm{Fe}_{14} \mathrm{~B}$ atomistic spin Hamiltonian, which was recently developed and successfully reproduced static properties of the $\mathrm{Nd}_{2} \mathrm{Fe}_{14} \mathrm{~B}$ magnet (shown in "Methods") ${ }^{25}$.

To calculate the free energy as a function of the $z$-component of the magnetization $F\left(M_{z}\right)$ for a huge system (up to $24.6 \mathrm{~nm} \times$ $24.6 \mathrm{~nm} \times 25.6 \mathrm{~nm}, 1,130,626$ spins), the size required to handle a nucleation process, we have developed an approach for the coercivity calculation based on the replica-exchange Wang-Landau (REWL) method ${ }^{26,27}$. Remarkably, we can perform an efficient parallelization scheme for the original Wang-Landau Monte Carlo (WLMC) method ${ }^{28}$ (see "Methods").

\section{RESULTS AND DISCUSSION}

Temperature dependence of the coercivity

The obtained $F\left(M_{\mathrm{z}}\right)$ for zero magnetic field $\left(H_{\mathrm{z}}=0\right)$ at $T=0.46 T_{\mathrm{C}}^{\mathrm{cal}}$ is depicted in Fig. 1a by the red curve ( $T_{C}^{\text {cal }}$ is the Curie temperature of the model). Notably, this is the quantitatively correct (not schematic) form of $F\left(M_{z}\right)$ for an atomistic spin model

\footnotetext{
${ }^{1}$ National Institute for Materials Science (NIMS), Tsukuba 305-0047, Japan. ${ }^{2}$ Elements Strategy Initiative Center for Magnetic Materials (ESICMM), Tsukuba, Japan. ${ }^{3}$ Institute for Solid State Physics, University of Tokyo, Kashiwa 277-8581, Japan. ${ }^{4}$ The Physical Society of Japan, Tokyo 113-0033, Japan. ${ }^{5}$ Department of Applied Physics, Tohoku University, Sendai 980-8579, Japan. ${ }^{6}$ National Institute of Advanced Industrial Science and Technology (AIST), Tsukuba 305-8568, Japan. ${ }^{凶}$ email: toga@issp.u-tokyo.ac.jp
} 
a
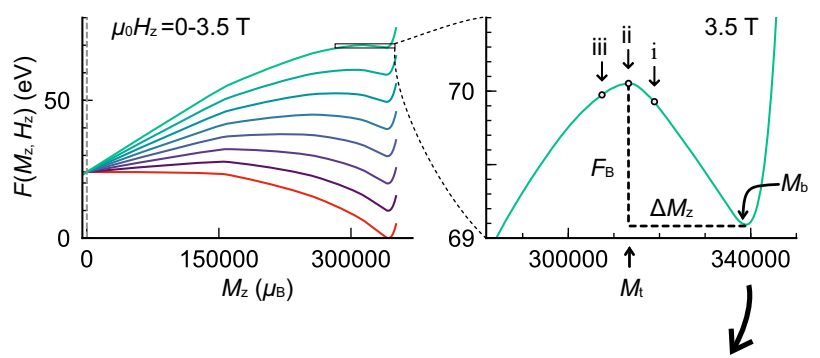

b

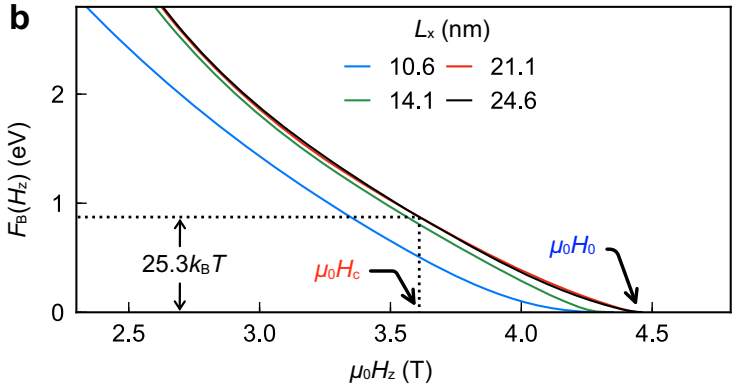

C

(i)

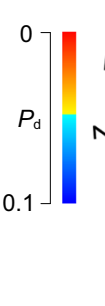

d

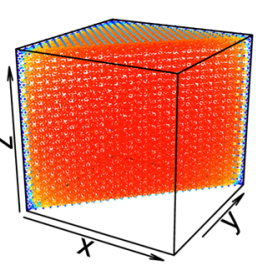

(i)

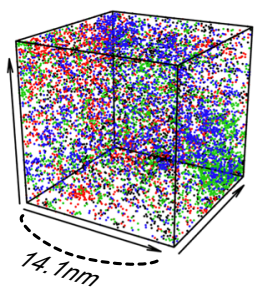

(ii)

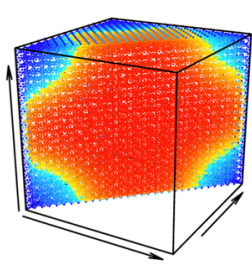

(ii)

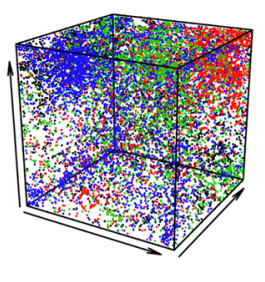

(iii)

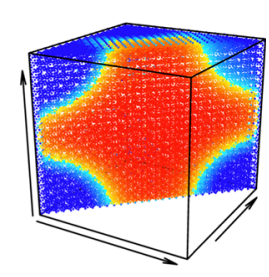

(iii)

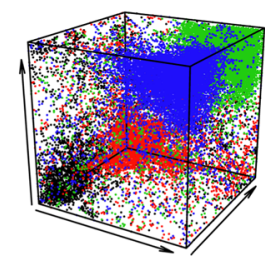

Fig. 1 Free energy landscape simulation. a Free energies as a function of $M_{z}$ for the $\mathrm{Nd}_{2} \mathrm{Fe}_{14} \mathrm{~B}$ isolated grains at $0.46 T_{C}^{\text {cal }}$, whose size is $\left(L_{x}, L_{y}\right.$, $\left.L_{z}\right)=(14.1,14.1,14.6) \mathrm{nm}\left(212,536\right.$ spins). Red line is for $H_{z}=0$ and other lines are for applying $H_{z}$. b Free-energy barriers as a function of $\mu_{0} H_{z}$ for four system sizes: $L_{x}=10.6,14.1,21.1$, and $24.6 \mathrm{~nm}\left(L_{y}=L_{x}, L_{z}=1.038 L_{x}\right)$, evaluated from $F\left(M_{z}, H_{z}\right)$ similar to Fig. 1a. c The distributions of reversed probability $P_{d}$ at Fe spins sliced by (110) plane, which correspond to the points (i-iii) in Fig. 1a. d Four MC snapshots of reversed Fe spins for the three points.

representing the $\mathrm{Nd}_{2} \mathrm{Fe}_{14} \mathrm{~B}$ magnet. Shapes of the free energy with the reverse magnetic fields $H_{z}$ are exactly given by $F\left(M_{z}\right)+\mu_{0} H_{z} M_{z}$. This is clearly illustrated in Fig. 1a. Close to the spinodal point, the point at which the metastability disappears, magnetization reversal occurs when the free energy barrier $F_{B}$ (see Fig. 1a) becomes compatible with the temperature. The $H_{z}$ dependence of $F_{B}$ is given in Fig. $1 \mathrm{~b}$, where we define $H_{0}$ as the field at which $F_{\mathrm{B}}=0$. Similarly, $H_{\mathrm{C}}$ is defined as the field at which $F_{\mathrm{B}}=25.3 k_{\mathrm{B}} T$. We call the former "spinodal field" and the latter "coercivity with thermal activation". As mentioned above, $H_{c}$ corresponds to the coercive field for the observation time $\tau=1.0 \mathrm{~s}$. In order to see how the magnetization reversal initiates, in Fig. 1c, we depict the spatial distributions of the magnetization reversal probability at the points shown by arrows in Fig. 1a, which were obtained by the REWL method. As expected, we found out that the reversal begins at the corner. There are two reasons for this reversal: One is the decrease of a local magnetic anisotropy due to surface thermal fluctuations. Secondly, the reduction of an exchange energy by decouplings at the corner facilitates the nucleation. Here, all the corners are equivalent, and symmetric configurations are found. However, in an individual process, one of the corners is selected. Figure $1 \mathrm{~d}$ illustrates a plot of $\mathrm{MC}$ snapshots at the corresponding fields.

The temperature dependencies of several formulae for the coercivities are depicted in Fig. 2, and are compared with the ideal coercivity $H_{\mathrm{k}}$. Here, we determined $H_{\mathrm{k}}$ as $H_{0}$ of the same system size with the periodic boundary condition. In the calculated temperature range, we confirmed that $H_{\mathrm{k}}$ takes almost same value as the magnetic anisotropy field $H_{a}=2 K_{1} / \mathcal{M}_{s}$, where $K_{1}$ is the magnetic anisotropy constant ${ }^{20,25}$ and $\mathcal{M}_{\mathrm{s}}$ is the saturated magnetization at the given temperature. The coercivity $H_{r}$ of permanent magnets is reduced from its limit value $H_{k}$ by various external influences. These reductions have been expressed in the following forms ${ }^{13,16}$ :

$$
\begin{aligned}
H_{r} & =a H_{\mathrm{k}}-H_{\mathrm{t}}-\mathcal{M}_{\mathrm{s}} N_{\mathrm{d}} \\
& =a^{\prime} H_{\mathrm{k}}-\mathcal{M}_{\mathrm{s}} N_{\mathrm{d}},
\end{aligned}
$$

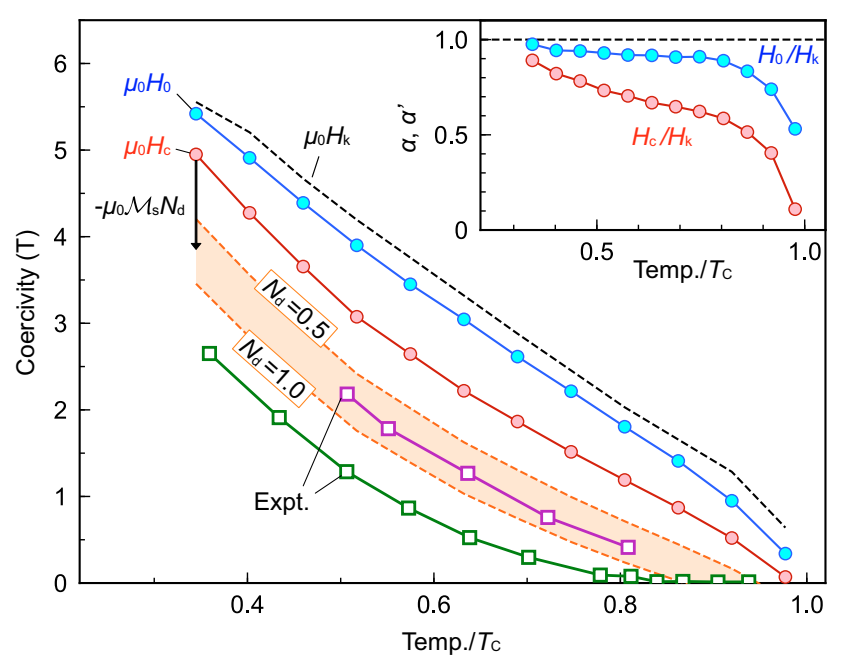

Fig. 2 Temperature dependence of coercivity. The blue line $\mu_{0} H_{0}$ and the red line $\mu_{0} H_{c}$ were calculated from Fig. $1 \mathrm{~b}$ for $21.1 \mathrm{~nm} \times 21.1$ $\mathrm{nm} \times 21.9 \mathrm{~nm}(713,172$ spins) isolated grain at each temperature. The colored area depicts the coercivity $\mu_{0} H_{c}$ under the demagnetization fields in the range of demagnetization factor $N_{d}=0.5-1.0$. The green and purple squared lines denote the experimental measurements in sintered ${ }^{29}$ and the hot-deformed with grain boundary diffusion of $\mathrm{Nd}-\mathrm{Cu}$ alloy ${ }^{14}$ magnets, respectively. Inset shows $a=H_{0} / H_{\mathrm{k}}$ and $a^{\prime}=H_{\mathrm{c}} / H_{\mathrm{k}}$.

where $H_{\mathrm{t}}=H_{0}-H_{\mathrm{c}}$ and $a=H_{0} / H_{\mathrm{k}}$. Here, $H_{\mathrm{t}}$ and $a$ represent the thermal activation field and phenomenological factor, respectively. The factor $a$ often means the reduction due to the microstructure such as defects, and the surfaces. On the other hand, $a \prime=H_{\mathrm{c}} / H_{\mathrm{k}}$ is an expression with an alternate definition for $H_{\mathrm{k}}$ including $H_{\mathrm{t}}$. The second line of Eq. 2 is known as "Kronmüller equation". The terms of the demagnetization field $-\mathcal{M}_{\mathrm{s}} N_{\mathrm{d}}$ express the reduction due to the dipole-dipole (DD) interaction. 

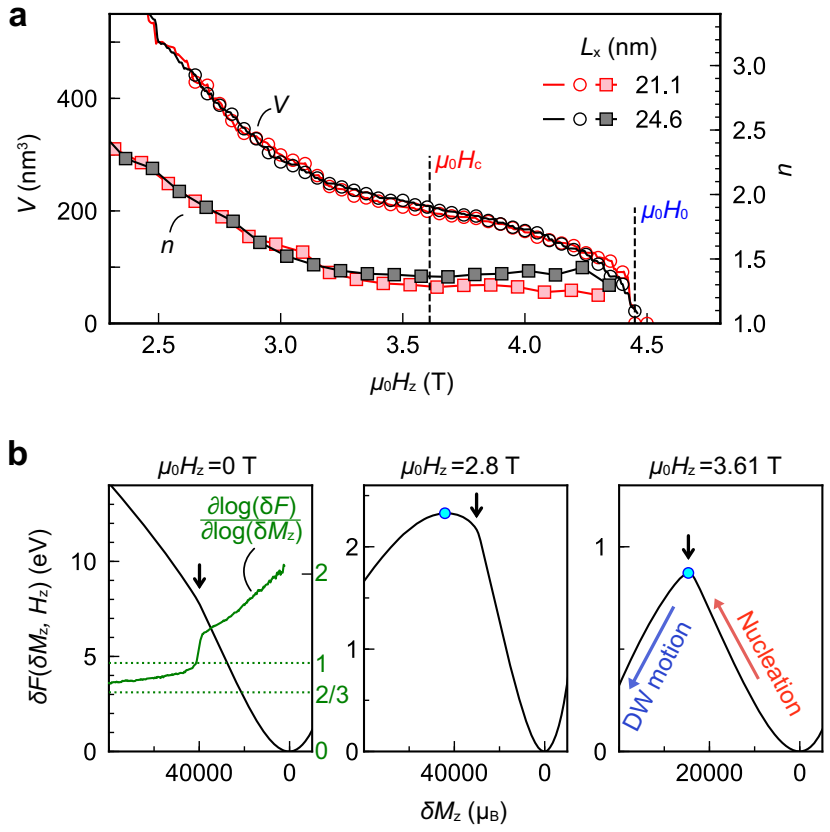

Fig. 3 Magnetic field dependence of activation volume. a The activation volume $V$ as a function of $\mu_{0} H_{z}$ for two system sizes at $0.46 T_{\mathrm{C}}^{\mathrm{cal}}$. Circle points are evaluated from Eq. 3 while solid lines are evaluated from Eq. 4. Squared lines are the exponent $n$ of free energy barrier in formula 6 . b Field dependence of the shape of free energy as a function of $\delta M_{z}$ for $L_{x}=24.6 \mathrm{~nm}$. Blue points are the top of the free energy barrier, which corresponds to $M_{\mathrm{t}}$, and black arrows denote the cusp in the free energy. Green line shows the value on the right axis.

Our approach can handle the temperature dependence of $a$ and $H_{\mathrm{t}}$ explicitly. Unlike the continuum approximation, temperature is naturally introduced in our model by the Boltzmann weights in the MC simulation. This is because we used the atomistic model. The continuum approximation works with temperaturedependent input parameters in which thermal fluctuations are not included in the motion. We have also plotted the results of coercivity $H_{\mathrm{r}}$ with respect to the demagnetization factors $N_{\mathrm{d}}=0.5$ and 1.0 with temperature-dependent magnetization $\mathcal{M}_{\mathrm{s}}$. Since the demagnetization field $-N_{\mathrm{d}} \mathcal{M}_{\mathrm{s}}$ approximately introduces the effects of the DD interaction as the uniform field, so it cannot consider the size and shape dependences of $N_{d}{ }^{30}$. However, the simulation results qualitatively validate those of the experiments because an increase in temperature leads to a downward convexity in coercivity even without $-N_{d} \mathcal{M}_{s}$. The effects of the DD interaction will be discussed later. In the figure, we compare the result with experimental observations. The green squares are data for a sintered sample and the purple ones for a diffused hotdeformed sample. In the former, the effect of polycrystalline causes a further reduction ${ }^{31}$, while in the latter grains are separated and compatible with the present calculation. In the former case, other effects would subject to the multi-grain approach in the future. Thus, the present result gives the upper limit of the coercivity at finite temperatures.

For some sintered polycrystalline magnets (corresponding to the green line in Fig. 2), Kronmüller and Durst phenomenologically estimated the decay factor as $a_{\text {exp }}^{\prime}=0.89-0.93$ from measured magnetic properties around room temperature ${ }^{16}$. In the inset of Fig. 2, we show the temperature dependence of $a$ and $a^{\prime}$. The difference of $a$ from 1 reflects the decrease of the surface magnetic anisotropy by thermal fluctuations, and $\alpha^{\prime}$ also includes the thermal activation effects. The decay factors at room temperature (here, $T=0.51 T_{\mathrm{C}}$ ) are determined as $a=0.93$ and $a^{\prime}=0.73$. In the case of the experiment, exchange interactions at the grain boundary interfaces suppress the interface thermal fluctuations. The suppression is one of the origins of the difference between $a_{\exp }^{\prime}$ and $\alpha^{\prime}$.

\section{Nucleation mechanism}

Next, we consider the mechanism of the thermal activation (nucleation) process for which the concept of "activation volume" has been introduced ${ }^{11-15}$. The symbol $\Delta M_{z}$ represents the difference of the magnetization between those at the local minimum $M_{\mathrm{z}}=M_{\mathrm{b}}$ of the free energy and those at the local maximum $M_{\mathrm{z}}=M_{\mathrm{t}}$ (see Fig. 1a). It is confirmed that $\Delta M_{\mathrm{z}}$ gives the activation volume $V$ :

$V=\Delta M_{\mathrm{z}} / \mathcal{M}_{\mathrm{s}}$.

Note that the unit of $\mathcal{M}_{\mathrm{s}}$ is ( $\mu_{\mathrm{B}}$ /volume). Activation volume has been defined by

$V=-\frac{1}{\mu_{0} \mathcal{M}_{\mathrm{s}}} \frac{\partial F_{\mathrm{B}}}{\partial H_{\mathrm{z}}}$

By differentiating $F_{\mathrm{B}}$ :

$$
\begin{aligned}
F_{\mathrm{B}}\left(H_{\mathrm{z}}\right) & =F\left(M_{\mathrm{t}}, H_{\mathrm{z}}\right)-F\left(M_{\mathrm{b}}, H_{\mathrm{z}}\right) \\
& =F\left(M_{\mathrm{t}}, 0\right)-F\left(M_{\mathrm{b}}, 0\right)+\mu_{0} H_{\mathrm{z}}\left(M_{\mathrm{t}}-M_{\mathrm{b}}\right),
\end{aligned}
$$

it is obvious that definition Eqs. 3 and 4 are equivalent. Here, we note that $F\left(M_{\mathrm{t}(b)}, 0\right)$ depends on $H_{\mathrm{z}}$ since $M_{\mathrm{t}(b)}$ is a function of $H_{\mathrm{z}}$. In Fig. $3 a$, the activation volumes obtained by the both definitions Eqs. 3 and 4 were plotted. This confirms the equivalence.

From Eq. 4, the field dependence of $F_{\mathrm{B}}$ is related to the nucleation process. Thus, we focus on the exponent $n$ which is widely used for the dependence in the phenomenological form:

$F_{\mathrm{B}} \propto\left(1-H_{\mathrm{z}} / H_{0}\right)^{n}$.

The exponent $n$ is 2 for coherent magnetization reversal. However, according to the authors ${ }^{12,14}$, the experimental value for $n$ was given to be approximately 1.0. We evaluated the value of $n$ in the atomistic magnetization reversal process around the coercivity. It should be noted that the value of $n$ varies depending on the range of $\mu_{0} H_{z}$ (shown in Fig. 3a), where we fit the data. However, for a wide range of $\mu_{0} H_{z}$ it was observed that the value of $n$ was approximately 1.3. We, therefore, propose that the small value of $n$ is ascribed to the peculiar shape of the free energy. Here we define $\delta F\left(\delta M_{\mathrm{z}}\right)$ for the free energy near the local minimum, i.e., $\delta F$ $\left(\delta M_{\mathrm{z}}\right)=F\left(M_{\mathrm{b}}-\delta M_{\mathrm{z}}\right)-F\left(M_{\mathrm{b}}\right)$, where $\delta F\left(\Delta M_{\mathrm{z}}\right)=F_{\mathrm{B}}$. The shapes of $\delta F\left(\delta M_{z}\right)$ for the fields $\mu_{0} H_{z}=0,2.8$, and $3.61 \mathrm{~T}$ are depicted in Fig. $3 \mathrm{~b}$, where a cusp is pointed by the arrow in each figure.

The cusp represents the point at which the type of the magnetization reversal changes ${ }^{32}$ : for small value of $\delta M_{z}$ (see in Fig. $3 b)$, the reversal process is the nucleation type, where we expect

$\delta F\left(\delta M_{z}\right) \propto \delta M_{z}$

while for large value of $\delta M_{z}, \delta F\left(\delta M_{z}\right)$ is mainly given by the change due to a domain wall (DW) motion, where we expect

$\delta F\left(\delta M_{z}\right) \propto \delta M_{z}^{2 / 3}+$ const.

This magnetization reversal mechanism is schematically pictured in Fig. 4. This difference can be understood in the following picture. In the case of the Heisenberg type with uniaxial anisotropy (corresponding to the $\mathrm{Nd}_{2} \mathrm{Fe}_{14} \mathrm{~B}$ spin model), the critical nucleation size $\left(\Delta M_{z}^{1 / 3} \equiv R_{c}\right)$ is about the domain wall width $\delta_{\mathrm{dw}}$. And thus, before the cusp point $\left(R<R_{\mathrm{c}}\right.$, where $R$ is the width of the magnetization reversing region, see Fig. $4 \mathrm{~b}$ ) the nucleation region is growing where we have Eq. 7. Around the cusp point, a fully reversed region appears. And after this point, the excess free energy $\delta F$ comes from the domain wall, i.e., 
a Heisenberg type

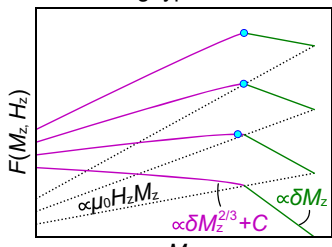

$M_{z}$

C Ising type

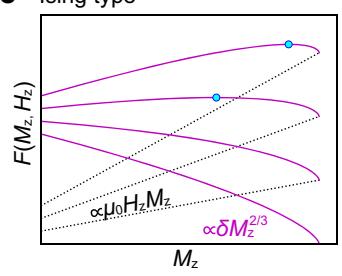

b

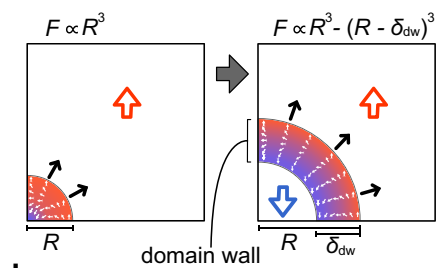

d

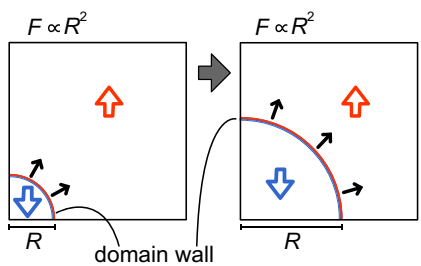

Fig. 4 Nucleation picture in permanent magnets. Schematic pictures of $\mathbf{a}$, $\mathbf{c}$ energy landscape and $\mathbf{b}$, $\mathbf{d}$ spin configuration in the magnetization reversal processes at low-temperature limit. a, b Heisenberg type spin model with uniaxial magnetic anisotropy. c, d lsing type. The energy landscapes under several magnetic fields are drawn (the lowest line is for $H_{z}=0$ ). Dotted lines are the reverse magnetic field terms $\mu_{0} H_{z} M_{z}$ and blue points are the top of the energy barriers.

the surface of the fully reversed region. In this case we have Eq. 8. For comparison, we show the case of Ising model where $\delta_{\mathrm{dw}}$ is much smaller than $R_{\mathrm{c}}$ at low temperatures, and we have the dependence Eq. 8 for almost all $R$ except very small $R$. This comparison is schematically pictured in Fig. $4 \mathrm{~d}$.

To clearly see the change of exponent, in Fig. $3 \mathrm{~b}$, we show the differentiate $\log (\delta \mathrm{F})$ by $\log \left(\delta \mathrm{M}_{\mathrm{z}}\right)$ with the green curve which gives roughly the power $k$ when we assume the dependence: $\delta F=C \delta M_{z}^{k}+D$ ( $C$ and $D$ are constant). We find that $k$ is close to 2/ 3 for large $\delta M_{z}$ and jump at the cusp. Around $\delta M_{z}=0$, thermal fluctuations enhance the behavior of $k=2$, i.e.,

$\delta F\left(\delta M_{\mathrm{z}}\right) \propto \delta M_{\mathrm{z}}^{2}$,

which makes it difficult to identify the behavior $\delta F \propto \delta M_{z}$ (i.e., $k=$ 1) for the nucleation process. This fact causes that we have an intermediate value ( $n \simeq 1.3$ ) numerically. However, $k$ clearly changes from 2 to 1 with $\delta M_{z}$, which supports the present scenario.

In any case, $\delta F$ has regions of upward convex and downward convex between which the cusp exists. This structure causes $\Delta M_{z}$ to be less dependent on $\mathrm{H}_{\mathrm{z}}$ for a range of $\mathrm{H}_{\mathrm{z}}{ }^{33}$, which can be seen from comparing the blue points in Fig. $4 a$, c. In this range, $V$ is a constant and it results in the value of $n=1$ by Eq. 4 . Note that, the reason for the cusp explains the drastic change in the magnetization distribution between (i) and (iii) in Fig. 1c, d.

If we assume $n=1$ for any $H_{z}$, then the result is the widely used phenomenological equation for the thermal activation effects ${ }^{13}$ :

$H_{\mathrm{t}}^{\prime}=\frac{25.3 k_{\mathrm{B}} T}{\mu_{0} \mathcal{M}_{\mathrm{s}} V_{\mathrm{c}}}$

where $V_{c}$ is the value of $V$ at $H_{z}=H_{c}$. In Fig. 5, we compare the temperature dependence of $H_{\mathrm{t}}$ and $H_{\mathrm{t}}^{\prime}$. We found a qualitatively similar dependence. The difference between $H_{\mathrm{t}}^{\prime}$ and $H_{\mathrm{t}}$ becomes significant in the high-temperature range, which is attributed to the fact that the activation volume and $n$ are not exactly constant. Thus, the difference due to the changes in the magnetization reversal type and surface effects, as shown in Figs. 3 and 4.

It is necessary to mention the exact effects of the DD interaction, which is approximately introduced as the

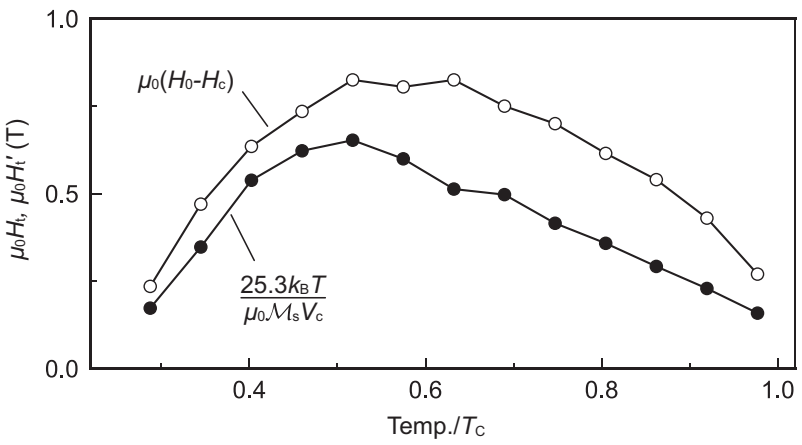

Fig. 5 Thermal activation reduction. The shifts of the coercivity due to the thermal activation effects, evaluated from the two ways: $H_{\mathrm{t}}=H_{0}-H_{c}$ and Eq. 10 .

demagnetization field in the present study. The DD interaction brings the non-uniformity of local demagnetization fields near corners and edges in grains, unlike the demagnetization field. The non-uniformity should change the nucleation process from the calculation results. However, the strength of the local field increases logarithmically with system size ${ }^{30,34,35}$, and the nucleation behavior is protected by the cusp structure. Thus, the nucleation mechanism we proposed is expected not to qualitatively change up to a certain large grain size $\left(L_{x} \simeq 1-10 \mu \mathrm{m}\right)$. Above this size, since magnetic domain structures are formed ${ }^{36}$, the DD interaction should be incorporated into the spin Hamiltonian.

In summary, our atomistic approach by the free energy landscape simulations revealed essential characteristics of the thermal activation effects for permanent magnets, i.e., the downward convexity in the coercivity concerning the temperature, the microscopic definition of the activation (nucleation) volume, and also the exponent $n=1$ for the well-used energy barrier formula (6). The present study should be the first step to study the characterization of coercivity at finite temperatures from microscopic information, and the method will be extended to the cases complex of grains with the DD interaction in the future.

\section{METHODS}

Atomistic spin model

The atomistic classical spin Hamiltonian of $\mathrm{Nd}_{2} \mathrm{Fe}_{14} \mathrm{~B}$ magnet was recently proposed for the study of thermodynamic properties at finite temperatures by using Monte Carlo (MC) method ${ }^{20,25,35,37}$ and stochastic Landau-Lifshitz-Gilbert equation ${ }^{38}$. It is given by

$$
\mathcal{H}=-2 \sum_{\mathrm{i}<\mathrm{j}} J_{\mathrm{ij}} \boldsymbol{s}_{\mathrm{i}} \cdot \boldsymbol{s}_{\mathrm{j}}-\sum_{\mathrm{i} \in \mathrm{Fe}} D_{\mathrm{i}}\left(s_{\mathrm{i}}^{z}\right)^{2}-\sum_{\mathrm{i} \in \mathrm{Nd}} \sum_{\mathrm{l}=2,4,6} D_{\mathrm{i}}^{\mathrm{l}}\left(s_{\mathrm{i}}^{z}\right)^{1},
$$

where $J_{\mathrm{ij}}$ is the exchange coupling constants between the $\mathrm{i}$-th and $\mathrm{j}$-th spins up to a cut-off range (in the present work $3.52 \AA$ ), and $s_{i}$ is the normalized spin moment at the i-th site. The second and third terms are the magnetic anisotropy of $\mathrm{Fe}$ and $\mathrm{Nd}$ sites, respectively. The coefficients $J_{\mathrm{i},}, D_{\mathrm{i}}, D_{\mathrm{i}}^{l}$ and magnetic moments are determined from ab-initio calculations and experimental data ${ }^{25}$. Note that Curie temperature of the model with given parameters is $T_{C}^{\mathrm{cal}}=870 \mathrm{~K}$, which is higher than the experimental observation $\left(T_{C}^{\exp } \simeq 585 \mathrm{~K}\right.$ ). This difference must be adjusted by finetuning of the parameters, but the qualitative properties are well reproduced $^{20,25}$.

Wang-Landau method for magnetization

The partition function of a spin system is generally described as follows:

$Z=\sum_{\boldsymbol{R}} \mathrm{e}^{-\beta H(\boldsymbol{R})}=\sum_{E} \sum_{M_{z}} g\left(E, M_{\mathrm{z}}\right) \mathrm{e}^{-\beta E}$,

where $\beta=1 / k_{B} T$ is the inverse temperature, $E$ is the internal energy of a spin configuration $R, M_{z}$ is the $z$-component of a total magnetization and $g$ 
$\left(E, M_{\mathrm{z}}\right)$ is the joint density of states. By using $g\left(E, M_{\mathrm{z}}\right)$, the magnetization dependence of the free energy $F\left(\beta, M_{z}\right)$ and a partial partition function $Z_{M_{z}}$ can be defined by following formula:

$F\left(\beta, M_{z}\right)=-\beta^{-1} \ln Z_{M_{z}}$,

$Z_{M_{\mathrm{z}}}=\sum_{E} g\left(E, M_{\mathrm{z}}\right) \mathrm{e}^{-\beta E}$.

The Wang-Landau Monte Carlo (WLMC) method $^{28}$ allows us to calculate $g$ $\left(E, M_{\mathrm{z}}\right)$ in the basis of a relation: $h\left(E, M_{\mathrm{z}}\right)=g\left(E, M_{\mathrm{z}}\right) w\left(E, M_{\mathrm{z}}\right)$, where $h\left(E, M_{\mathrm{z}}\right)$ is the histogram of the spin states obtained by MC sampling with a weight $W$ $\left(E, M_{z}\right)$. If a flat histogram ( $h$ is constant) can be obtained by arbitrarily adjusting $w\left(E, M_{\mathrm{z}}\right)$ in a $\mathrm{MC}$ simulation, $g\left(E, M_{\mathrm{z}}\right)$ is proportional to the reciprocal of the adjusted $w\left(E, M_{z}\right)$

As mentioned above, adjusting the weight for two-dimensional or multidimensional space requires massive calculation cost. To reduce the cost, for the MC sampling in the energy space, we use Boltzmann weight, i.e., $w\left(E, M_{z}\right)=w\left(M_{z}\right) \exp (-\beta E)^{39,40}$. Then, the histogram of $M_{z}$ space can be written as follows (using Eq. 14):

$$
\begin{aligned}
h\left(M_{\mathrm{z}}\right) & =\sum_{E} h\left(E, M_{\mathrm{z}}\right) \\
& =w\left(M_{\mathrm{z}}\right) Z_{M_{\mathrm{z}}} .
\end{aligned}
$$

Therefore, by obtaining a flat histogram in one dimensional space of $M_{z}$ to adjust $w\left(M_{z}\right)$, the free energy can be calculated from Eq. 13:

$$
\begin{aligned}
F\left(\beta, M_{\mathrm{z}}\right) & =-\beta^{-1} \ln \frac{C}{w_{\mathrm{f}}\left(M_{\mathrm{z}}\right)} \\
& =C+\beta^{-1} \ln w_{\mathrm{f}}\left(M_{\mathrm{z}}\right),
\end{aligned}
$$

where $C$ is constant, $w_{\mathrm{f}}$ is the adjusted $w$ when obtaining the flat histogram. Since the continuous spin system cannot determine $C$ by calculation at each temperature, so in this study, we proceed with the calculation of the coercivity using the relative values of $F$. Although the above scheme requires the simulation for each temperature due to the use of Boltzmann weight, it significantly reduces the calculation cost for free energy as a function of $M_{z}$.

\section{$1 / \mathrm{t}$ algorithm}

To obtain a flat histogram, the WLMC method adjusts the weight as $w\left(M_{\mathrm{z}}^{\prime}\right) \rightarrow \mathrm{e}^{-\eta} w\left(M_{\mathrm{z}}^{\prime}\right)$ every update attempt (even if no update) of the spin state with a random walk, where $\eta(>0)$ is a modification factor, $M_{z}^{\prime}$ is the total magnetization of the state after the attempt. From the formulations of the previous section, update attempts of the spin state were performed according to the following transition probability:

$$
\begin{aligned}
P\left(E, M_{\mathrm{z}} \rightarrow E^{\prime \prime}, M_{\mathrm{z}}^{\prime \prime}\right) & =\min \left(1, \frac{w\left(E^{\prime \prime}, M_{\mathrm{z}}^{\prime \prime}\right)}{w\left(E, M_{\mathrm{z}}\right)}\right) \\
& =\min \left(1, \mathrm{e}^{-\beta\left[E^{\prime \prime}-E-G\left(M_{\mathrm{z}}^{\prime \prime}\right)+G\left(M_{\mathrm{z}}\right)\right]}\right), \\
G\left(M_{\mathrm{z}}\right) & =\beta^{-1} \ln w\left(M_{\mathrm{z}}\right),
\end{aligned}
$$

where $E^{\prime \prime}$ and $M_{z}^{\prime \prime}$ are the values of a trial state. Therefore, in the actual simulation, $G$ is adjusted instead of $w$ as $G\left(M_{\mathrm{z}}^{\prime}\right) \rightarrow G\left(M_{\mathrm{z}}^{\prime}\right)-\tilde{\eta}$, where $\tilde{\eta}=$ $\beta^{-1} \eta(>0)$. This redefined modification factor $\tilde{\eta}$ has the unit of energy, which is convenient to adjust $G$ with consistent values regardless of temperature.

Adjustment of $G$ at every update attempt accelerates the convergence; however, it breaks a detailed balance condition. Thus, it is necessary to reduce $\tilde{\eta}$ so as not to affect the results of the MC simulation. To perform the reduction, the present study adopt the so-called $1 / t$ algorithm ${ }^{41}$ which resolves systematic error due to histogram flatness condition in the original WLMC method. The algorithm reduces the $\tilde{\eta}$ as $a N_{\text {bin }} / t$, where $a$ is proportionality coefficient (here, we set $a=1.0 \mathrm{eV}$ ), $t$ is the number of update attempts of the spin state and $N_{\mathrm{bin}}$ is the number of $M_{\mathrm{z}}$ levels in the simulation range of interest, $M_{\mathrm{z}} \in\left[M_{\mathrm{z}}^{\min }, M_{\mathrm{z}}^{\max }\right]$. Note that, since the WLMC method requires to treat $M_{z}$ discretely for the $M C$ sampling with Eq. 17, in this study, we adopt the grid where $h\left(M_{z}\right)$ and $G\left(M_{z}\right)$ are discretized with the bins of $m_{\mathrm{w}}=0.3-0.5 \mu_{\mathrm{B}}$ width, and $N_{\mathrm{bin}}=\left(M_{\mathrm{z}}^{\max }-M_{\mathrm{z}}^{\min }\right) / m_{w}$. As a practical matter, during small $t$, the changes in $\tilde{\eta}$ is so large that the simulation does not convergence. Thus, the initial value of $\tilde{\eta}$ is set to be 1.0 $\mathrm{eV}$, and it is reduced until $\tilde{\eta} \leq N_{\text {bin }} / t(\mathrm{eV})$ as $\tilde{\eta} \rightarrow \tilde{\eta} / 2$ at every random walker visit to the all the magnetization bins.
Algorithm 1.

The REWL method for $M_{z}$ with $1 / t$ algorithm.

Set spin model \& parallelization

$$
\begin{aligned}
& t=0 ; h(:)=0 ; G(:)=0.0 ; \tilde{\eta}=1.0 ; \alpha=1.0 \\
& \text { for } \\
& \text { for i-site=1: } N_{s} \\
& \text { Update i-site using Eq. (17) } \\
& \text { Calculate } M_{z} \\
& t+=1 \\
& \text { end } \\
& h\left(M_{z}\right)+=1 ; G\left(M_{z}\right)=\tilde{\eta} \\
& h\left(M_{\mathrm{z}}\right)+=1 ; G\left(M_{\mathrm{z}}\right)=\tilde{\eta}
\end{aligned}
$$

Replica connection for $G \& h$

end

Check convergence, if ( true ): exit

Output $G$ as free energy $F$

Replica exchange parallelization approach

For further large-scale simulation, we use the replica-exchange Wang-Landau (REWL) method ${ }^{26,27}$, which is an efficient parallelization approach. The simple idea for parallelization in the WLMC method can be realized to divide the magnetization range by a small range and to allocate the piece into each processor. Each processor simulates the WLMC method individually. However, the small range may break ergodicity and inhibit the relaxation of the spin state. The REWL method is to recover the ergodicity by exchanging the spin configurations among these processors.

For the replica-exchange (RE), it is necessary to overlap the areas allocated to each processor, like Fig. 6. Previous research ${ }^{27}$ proposed that the efficiently overlap ratio is $62.5-75 \%$ (we set $70 \%$ ). In this study, every $N_{\mathrm{s}}$ spin update attempt, we try to exchange the spin configuration between neighbor processors according to the following exchange probability:

$$
\begin{aligned}
P_{R}(X \in n \leftrightarrow Y \in m) & =\min \left(1, \frac{w_{\mathrm{n}}(Y) w_{\mathrm{m}}(X)}{w_{\mathrm{n}}(X) w_{\mathrm{m}}(Y)}\right) \\
& =\min \left(1, \mathrm{e}^{-\beta\left[G_{\mathrm{n}}(X)+G_{\mathrm{m}}(Y)-G_{\mathrm{n}}(Y)-G_{\mathrm{m}}(X)\right]}\right),
\end{aligned}
$$

where $n(, m)$ is the index of the adjacent processors, $X(, Y)$ is the spin configuration from which $E$ and $M_{\mathrm{z}}$ can be calculated, and $w_{n}\left(, G_{n}\right)$ is the values of $w(, G)$ on the processor $n$. The exchange probability only depends on $M_{\mathrm{z}}$ and not on $E$. If the spin configurations are not in the overlapping range, $P_{\mathrm{R}}=0$.

As mentioned in Eq. 16, $F$ (and $G$ ) can only be calculated as relative values. Thus, the values of $F$ for the processors require to be corrected and connected so that the average value of the overlapping range is equal. After this connection, the average of $F$ for all the processors is calculated as a result. Note that, just to improve connectivity among the processors, we 


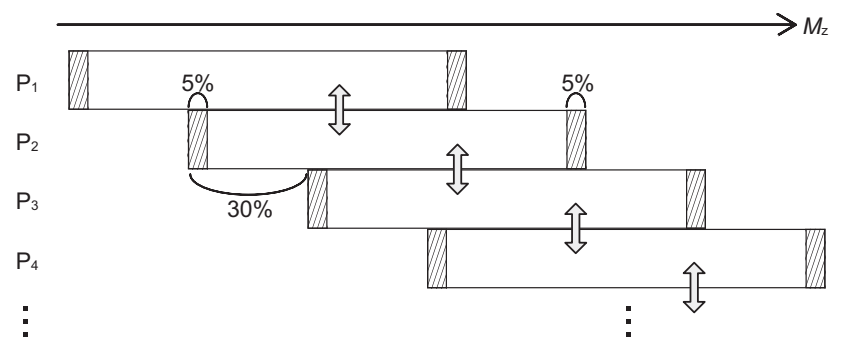

Fig. 6 Diagram of parallelization for the REWL method. The calculating range of the magnetization $M_{z}$ is partitioned by many processors, here $P_{1-4}$. The double-headed arrows mean the replicaexchange and the shaded ranges denote the area to be omitted when connecting $G$ among the processors (see text).

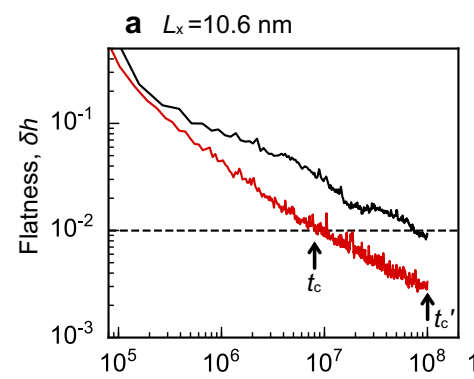

b $L_{x}=14.1 \mathrm{~nm}$

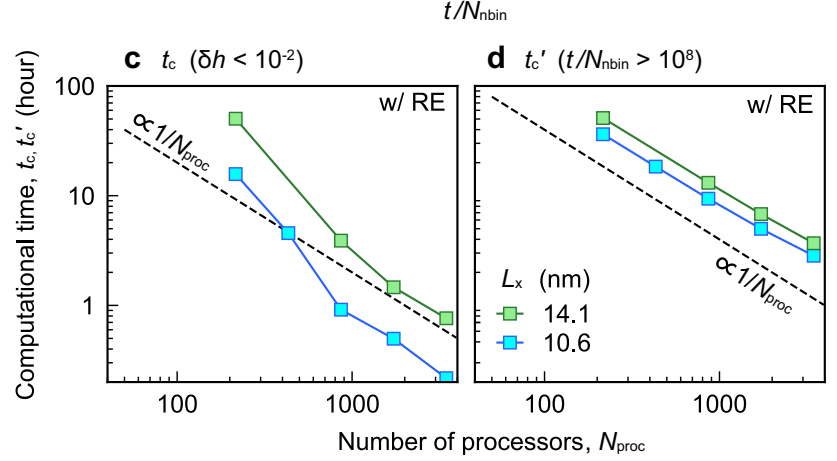

Fig. 7 Parallelization efficiency of replica exchange. The flatness $\delta H$ as a function of $t / N_{\text {bin }}(=1 / \tilde{\eta}(1 / \mathrm{eV}))$ at $0.46 T_{\mathrm{C}}^{\text {cal }}$ of the $\mathrm{Nd}_{2} \mathrm{Fe}_{14} \mathrm{~B}$ spin model with the isolated grain for two sizes a $L_{x}=10.6 \mathrm{~nm}$ and b $L_{x}=14.1 \mathrm{~nm}$. Black (Blue) lines are the results without (with) RE, which are performed by 864 processors. In the simulations, the calculated ranges of the magnetization are $M_{\mathrm{z}} \in\left[-0.02 \mathrm{M}_{\mathrm{z}}, 0.76 \mathrm{M}_{\mathrm{z}}^{\mathrm{t}}\right]$ for $L_{\mathrm{x}}=10.6 \mathrm{~nm}$ and $M_{\mathrm{z}} \in\left[0.3 M_{\mathrm{z}}^{\mathrm{t}}, 0.76 M_{\mathrm{z}}^{\mathrm{t}}\right]$ for $L_{\mathrm{x}}=14.1 \mathrm{~nm}$ with $m_{\mathrm{w}}$ $=0.5 \mu_{\mathrm{B}}$, where $M_{\mathrm{z}}^{\mathrm{t}}$ is the maximum value of $M_{\mathrm{z}}$ in each spin system. $\mathrm{C}$ $t_{c}$ and $\mathbf{d} t_{c}^{\prime}$ are the computational times as a function of the number of processors $N_{\text {proc }}$ which satisfy the convergence conditions $\delta h<$ $10^{-2}$ and $t / N_{\text {nbin }}>10^{8}$, respectively. Arrows in $\mathbf{a}$ and $\mathbf{b}$ denote the points corresponding to $t_{\mathrm{c}}$ and $t_{\mathrm{c}}^{\prime}$.

omit the edge ranges (5\%, shown in Fig. 6) of each processor in the above correction and averaging (not so important).

To show the effectiveness of the RE, in Fig. $7 a$, b, we plot the flatness $\delta h=[\max (h)-\min (h)] / \operatorname{mean}(h)$ as a function of $t / N_{\text {bin }}(=1 / \tilde{\eta})$ in the simulations with and without RE. The accuracy of the REWL method is determined by the smallness of $\delta H$ and $\tilde{\eta}$. Thus, the figure clearly indicates that $\mathrm{RE}$ is necessary to obtain a converged result, especially at large system size. The results in this study are set to satisfy the following convergence conditions: $\delta H<10^{-2}$ and $\tilde{\eta}<10^{-8}(\mathrm{eV})$. Figure $7 \mathrm{c}$ and $\mathrm{d}$ show the parallelization efficiency for the two system sizes in strong scaling with under the two convergence conditions. This result indicates that the parallelization is achieved with the ideal efficiency.
The pseudocode of the implemented methods in the present study is described in Algorithm 1. Through the methods, for large system size (up to $1,130,626$ spins), we made it possible to calculate the free energy as a function of $M_{z}$.

\section{DATA AVAILABILITY}

The data reported in this paper is available from the corresponding author upon reasonable request.

\section{CODE AVAILABILITY}

The calculation codes used in this paper is available from the corresponding author upon reasonable request.

Received: 3 December 2019; Accepted: 10 April 2020;

Published online: 01 June 2020

\section{REFERENCES}

1. Gutfleisch, O. et al. Magnetic materials and devices for the 21st century: stronger, lighter, and more energy efficient. Adv. Mater. 23, 821-842 (2011).

2. Chu, S. \& Majumdar, A. Opportunities and challenges for a sustainable energy future. Nature 488, 294-303 (2012).

3. Moriya, H., Tsuchiura, H. \& Sakumav, A. First principles calculation of crystal field parameter near surfaces of $\mathrm{Nd}_{2} \mathrm{Fe}_{14}$ B. J. Appl. Phys. 105, 07A740 (2009).

4. Hrkac, G. et al. The role of local anisotropy profiles at grain boundaries on the coercivity of Nd2Fe14B magnets. Appl. Phys. Lett. 97, 232511 (2010).

5. Mitsumata, C., Tsuchiura, H. \& Sakuma, A. Model calculation of magnetization reversal process of hard magnet in $\mathrm{Nd}_{2} \mathrm{Fe}_{14} \mathrm{~B}$ system. Appl. Phys. Express 4, 113002 (2011).

6. Suess, D. et al. Calculation of coercivity of magnetic nanostructures at finite temperatures. Phys. Rev. B 84, 224421 (2011).

7. Hono, K. \& Sepehri-Amin, H. Strategy for high-coercivity Nd-Fe-B magnets. Scr. Mater. 67, 530-535 (2012).

8. Suzuki, M. et al. Magnetic domain evolution in Nd-Fe-B:Cu sintered magnet visualized by scanning hard X-ray microprobe. Acta Mater. 106, 155-161 (2016).

9. Miyashita, S. et al. Perspectives of stochastic micromagnetism of $\mathrm{Nd}_{2} \mathrm{Fe}_{14} \mathrm{~B}$ and computation of thermally activated reversal process. Scr. Mater. 154, 259-265 (2018).

10. Miyake, T. \& Akai, H. Quantum theory of rare-earth magnets. J. Phys. Soc. Jpn. 87, 041009 (2018).

11. Gaunt, P. Magnetic viscosity and thermal activation energy. J. Appl. Phys. 59, 4129-4132 (1986)

12. Givord, D., Lienard, A., Tenaud, P. \& Viadieu, T. Magnetic viscosity in Nd-Fe-B sintered magnets. J. Magn. Magn. Mater. 67, L281-L285 (1987).

13. Givord, D., Lu, Q., Rossignol, M. F., Tenaud, P. \& Viadieu, T. Experimental approach to coercivity analysis in hard magnetic materials. J. Magn. Magn. Mater. 83, 183-188 (1990).

14. Okamoto, S. et al. Temperature-dependent magnetization reversal process and coercivity mechanism in Nd-Fe-B hot-deformed magnets. J. Appl. Phys. 118, 223903 (2015).

15. Bance, S. et al. Thermal activation in permanent magnets. JOM 67, 1350-1356 (2015).

16. Kronmüller, H., Durst, K. D. \& Sagawa, M. Analysis of the magnetic hardening mechanism in RE-FeB permanent magnets. J. Magn. Magn. Mater. 74, 291-302 (1988).

17. Givord, D., Rossignol, M. \& Barthem, V. M. T. S. The physics of coercivity. J. Magn. Magn. Mater. 258-259, 1-5 (2003).

18. Fischbacher, J. et al. On the limits of coercivity in permanent magnets. Appl. Phys. Lett. 111, 072404 (2017)

19. Fischbacher, J. et al. Searching the weakest link: demagnetizing fields and magnetization reversal in permanent magnets. Scr. Mater. 154, 253-258 (2018).

20. Toga, Y., Nishino, M., Miyashita, S., Miyake, T. \& Sakuma, A. Anisotropy of exchange stiffness based on atomic-scale magnetic properties in the rare-earth permanent magnet $\mathrm{Nd}_{2} \mathrm{Fe}_{14}$ B. Phys. Rev. B 98, 054418 (2018).

21. Gong, Q., Yi, M. \& Xu, B.-X. Multiscale simulations toward calculating coercivity of Nd-Fe-B permanent magnets at high temperatures. Phys. Rev. Mater. 3, 084406 (2019).

22. Zhang, L., Ren, W., Samanta, A. \& Du, Q. Recent developments in computational modelling of nucleation in phase transformations. NPJ Comput. Mater. 2, 16003 (2016). 
23. Dittrich, R. et al. A path method for finding energy barriers and minimum energy paths in complex micromagnetic systems. J. Magn. Magn. Mater. 250, 12-19 (2002).

24. Herbst, J. F. R2Fe14B materials: intrinsic properties and technological aspects. Rev. Mod. Phys. 63, 819-898 (1991).

25. Toga, Y. et al. Monte Carlo analysis for finite-temperature magnetism of $\mathrm{Nd}_{2} \mathrm{Fe}_{14}$ B permanent magnet. Phys. Rev. B 94, 219901-219901 (2016).

26. Nogawa, T., Ito, N. \& Watanabe, H. Evaporation-condensation transition of the two-dimensional Potts model in the microcanonical ensemble. Phys. Rev. E 84, 061107 (2011).

27. Vogel, T., Li, Y. W., Wüst, T. \& Landau, D. P. Generic, hierarchical framework for massively parallel Wang-Landau sampling. Phys. Rev. Lett. 110, 210603 (2013).

28. Wang, F. \& Landau, D. P. Efficient, multiple-range random walk algorithm to calculate the density of states. Phys. Rev. Lett. 86, 2050-2053 (2001).

29. Hirosawa, S. et al. The dependence of coercivity on anisotropy field in sintered RFe-B permanent magnets. J. Magn. Magn. Mater. 61, 363-369 (1986).

30. Grönefeld, M. \& Kronmüller, H. Calculation of strayfields near grain edges in permanent magnet material. J. Magn. Magn. Mater. 80, 223-228 (1989).

31. Mohakud, S., Andraus, S., Nishino, M., Sakuma, A. \& Miyashita, S. Temperature dependence of the threshold magnetic field for nucleation and domain wall propagation in an inhomogeneous structure with grain boundary. Phys. Rev. B 94, 054430 (2016)

32. Vries, Jd., Bolhuis, T. \& Abelmann, L. Temperature dependence of the energy barrier and switching field of sub-micron magnetic islands with perpendicular anisotropy. N. J. Phys. 19, 093019 (2017).

33. Skomski, R., Zhou, J., Kirby, R. D. \& Sellmyer, D. J. Micromagnetic energy barriers. J. Appl. Phys. 99, 08 B906 (2006).

34. Bance, S. et al. Grain-size dependent demagnetizing factors in permanent magnets. J. Appl. Phys. 116, 233903 (2014)

35. Hinokihara, T., Nishino, M., Toga, Y. \& Miyashita, S. Exploration of the effects of dipole-dipole interactions in $\mathrm{Nd}_{2} \mathrm{Fe}_{1} 4 \mathrm{~B}$ thin films based on a stochastic cutoff method with a novel efficient algorithm. Phys. Rev. B 97, 104427 (2018).

36. Billington, $D$. et al. Unmasking the interior magnetic domain structure and evolution in Nd-Fe-B sintered magnets through high-field magnetic imaging of the fractured surface. Phys. Rev. Mater. 2, 104413 (2018).

37. Nishino, M. et al. Atomistic-model study of temperature-dependent domain walls in the neodymium permanent magnet $\mathrm{Nd}_{2} \mathrm{Fe}_{14}$ B. Phys. Rev. B 95, 094429 (2017).

38. Nishino, M. \& Miyashita, S. Nontrivial temperature dependence of ferromagnetic resonance frequency for spin reorientation transitions. Phys. Rev. B 100, 020403 (2019).

39. Berg, B. A., Hansmann, U. \& Neuhaus, T. Simulation of an ensemble with varying magnetic field: a numerical determination of the order-order interface tension in the $D=2$ Ising model. Phys. Rev. B 47, 497-500 (1993).

40. Watanabe, K. \& Sasaki, M. An efficient Monte-Carlo method for calculating free energy in long-range interacting systems. J. Phys. Soc. Jpn 80, 093001 (2011).

41. Belardinelli, R. E. \& Pereyra, V. D. Fast algorithm to calculate density of states. Phys. Rev. E 75, 046701 (2007).

\section{ACKNOWLEDGEMENTS}

We acknowledge fruitful discussions with Satoshi Okamoto, Munetaka Sasaki, Masamichi Nishino, Taichi Hinokihara, and Satoshi Hirosawa. This work is partially supported by ESICMM, Grant Number JPMXP0112101004, through MEXT; by MEXT as a social and scientific priority issue (CDMSI) to be tackled by using a post-K computer The computation was performed on Supercomputers at NIMS; ISSP, University of Tokyo; ACCMS, Kyoto University; RIIT, Kyushu University.

\section{AUTHOR CONTRIBUTIONS}

Y.T. and T.M. conceived and designed the basic research plan. A.S. proposed the plan from the viewpoint of the activation volume. Y.T. and S.M. discussed and developed the free-energy calculation approach for the coercivity. Y.T. performed the free energy and the coercivity calculation. Y.T., S.M., and T.M. wrote the paper. All authors discussed the results.

\section{COMPETING INTERESTS}

The authors declare no competing interests.

\section{ADDITIONAL INFORMATION}

Correspondence and requests for materials should be addressed to Y.T.

Reprints and permission information is available at http://www.nature.com/ reprints

Publisher's note Springer Nature remains neutral with regard to jurisdictional claims in published maps and institutional affiliations.

Open Access This article is licensed under a Creative Commons Attribution 4.0 International License, which permits use, sharing, adaptation, distribution and reproduction in any medium or format, as long as you give appropriate credit to the original author(s) and the source, provide a link to the Creative Commons license, and indicate if changes were made. The images or other third party material in this article are included in the article's Creative Commons license, unless indicated otherwise in a credit line to the material. If material is not included in the article's Creative Commons license and your intended use is not permitted by statutory regulation or exceeds the permitted use, you will need to obtain permission directly from the copyright holder. To view a copy of this license, visit http://creativecommons. org/licenses/by/4.0/.

(c) The Author(s) 2020 